\title{
On the de Rham cohomology of solvmanifolds
}

\author{
Sergio Console And Anna Fino
}

\begin{abstract}
Using results by D. Witte [35] on the superigidity of lattices in solvable Lie groups we get a new proof of a recent remarkable result obtained by D. Guan [15] on the de Rham cohomology of a compact solvmanifold, i.e., of a quotient of a connected and simply connected solvable Lie group $G$ by a lattice $\Gamma$. This result can be applied to compute the Betti numbers of a compact solvmanifold $G / \Gamma$ even in the case that the solvable Lie group $G$ and the lattice $\Gamma$ do not satisfy the Mostow condition.
\end{abstract}

Mathematics Subject Classification (2010): 53C30 (primary); 22E25, 22E40 (secondary).

\section{Introduction}

Let $M$ be a compact solvmanifold, i.e. a quotient of a connected and simply connected solvable Lie group $G$ by a lattice $\Gamma$. Denote by $\operatorname{Ad}_{G}(G)$ (respectively, $\left.\operatorname{Ad}_{G}(\Gamma)\right)$ the subgroup of GL( $\left.\mathfrak{g}\right)$ generated by $e^{\operatorname{ad}_{X}}$, for all $X$ in the Lie algebra $\mathfrak{g}$ of $G$ (respectively, in the Lie algebra of $\Gamma$ ). It is well known that if $G$ is a simply connected solvable Lie group, then $\operatorname{Ad}_{G}(G)$ is a solvable algebraic group and $\operatorname{Aut}(G) \cong \operatorname{Aut}(\mathfrak{g})$. We will denote by $\mathcal{A}\left(\operatorname{Ad}_{G}(G)\right)$ and $\mathcal{A}\left(\operatorname{Ad}_{G}(\Gamma)\right)$ the real algebraic closures of $\operatorname{Ad}_{G}(G)$ and $\operatorname{Ad}_{G}(\Gamma)$ respectively.

In general, as a consequence of the Borel density theorem (see [34, Corollary 4.2] and Theorem 3.1 here) applied to the adjoint representation, one has that if $\Gamma$ is a lattice of a connected solvable Lie group $G$, then $\mathcal{A}\left(\operatorname{Ad}_{G}(G)\right)=T_{\text {cpt }} \mathcal{A}\left(\operatorname{Ad}_{G}(\Gamma)\right)$ is a product of the groups $T_{\mathrm{cpt}}$ and $\mathcal{A}\left(\operatorname{Ad}_{G}(\Gamma)\right)$, where $T_{\mathrm{cpt}}$ is any maximal compact torus of $\mathcal{A}\left(\operatorname{Ad}_{G}(G)\right)$.

If $\mathcal{A}\left(\operatorname{Ad}_{G}(G)\right)=\mathcal{A}\left(\operatorname{Ad}_{G}(\Gamma)\right)$, i.e., if $G$ and $\Gamma$ satisfy the Mostow condition, then the de Rham cohomology $H_{\mathrm{dR}}^{*}(M)$ of the compact solvmanifold $M=G / \Gamma$ can be computed by the Chevalley-Eilenberg cohomology $H^{*}(\mathfrak{g})$ of the Lie algebra $\mathfrak{g}$ of $G$ (see [26] and [30, Corollary 7.29]); indeed, one has the isomorphism

$$
H_{\mathrm{dR}}^{*}(M) \cong H^{*}(\mathfrak{g}) .
$$

This work was supported by the Projects MIUR "Riemannian Metrics and Differentiable Manifolds", "Geometric Properties of Real and Complex Manifolds" and by GNSAGA of INdAM.

Received December 24, 2009; accepted in revised form July 15, 2010. 
In the special case that $G$ is nilpotent, or equivalently that $M=G / \Gamma$ is a nilmanifold, the Lie group $\operatorname{Ad}_{G}(G)$ is unipotent and therefore its real algebraic closure $\mathcal{A}\left(\operatorname{Ad}_{G}(G)\right)$ coincides with $\operatorname{Ad}_{G}(G)$. Moreover, the real algebraic closure $\mathcal{A}\left(\operatorname{Ad}_{G}(\Gamma)\right)$ of the lattice $\operatorname{Ad}_{G}(\Gamma)$ coincides with $\operatorname{Ad}_{G}(G)$. For nilmanifolds the isomorphism (1.1) was previously shown by K. Nomizu [27] using the Leray-Serre spectral sequence. A similar isomorphism for the Dolbeault cohomology in the case of some classes of complex structures on nilmanifolds was proved in [7,8,31].

Nilpotent Lie groups are a subclass of solvable Lie groups, called the completely solvable ones. We recall that a solvable Lie group $G$ is completely solvable if all the linear operators $\operatorname{ad}_{X}: \mathfrak{g} \rightarrow \mathfrak{g}$ have only real eigenvalues (zero eigenvalues in the nilpotent case), for all $X \in \mathfrak{g}$. For nilpotent, or more generally completely solvable, Lie groups $G$, any lattice $\Gamma$ of $G$ satisfies the Mostow condition, so (1.1) holds and the isomorphism was first proved by A. Hattori [19] using a different method.

In the case where $\mathcal{A}\left(\operatorname{Ad}_{G}(G)\right) \neq \mathcal{A}\left(\operatorname{Ad}_{G}(\Gamma)\right)$, it is in general very difficult to compute the de Rham cohomology of $G / \Gamma$. As far as we know, the only known result has been obtained recently by D. Guan and it has been applied to the classification of compact complex homogeneous manifolds with pseudo-Kähler structures (see $[15$, main theorem 1] and $[16,17]$ ). He proved that, if $M=G / \Gamma$ is a compact solvmanifold, then there exists a finite covering space $\tilde{M}=G / \tilde{\Gamma}$, i.e., $\Gamma / \tilde{\Gamma}$ is a finite group, such that $\tilde{M}=\tilde{G} / \tilde{\Gamma}$, where $\tilde{G}$ is another simply connected solvable real Lie group $\tilde{G}$, diffeomorphic to $G$, and satisfiying the Mostow condition

$$
\mathcal{A}\left(\operatorname{Ad}_{\tilde{G}}(\tilde{G})\right)=\mathcal{A}\left(\operatorname{Ad}_{\tilde{G}}(\tilde{\Gamma})\right) .
$$

Guan proved the previous theorem by using the results of V. V. Gorbatsevich [11, 12] on Malcev and algebraic splitting of a solvable Lie group. The construction of the Malcev splitting (also called semisimple splitting) was introduced by A. Malcev [20] and later improved by L. Auslander [1]. The general idea consists in embedding solvable and general simply connected Lie groups into split groups, i.e., in groups which are representable as semidirect products $T \ltimes N$, where $N$ is the nilradical and $T$ is an Abelian subgroup acting on $N$ by semisimple automorphisms.

The nilradical of the semisimple splitting is called in the literature the nilshadow. The traditional nilshadow construction (see also [3]) kills an entire maximal torus. D. Witte in [35] refined the previous construction and he introduced a method for killing a specific compact subtorus of the maximal torus.

Using this result of Witte, we find a different proof of Guan's theorem. More precisely, we show the following:

Main theorem. Let $M=G / \Gamma$ be a compact solvmanifold, quotient of a simply connected solvable Lie group $G$ by a lattice $\Gamma$, and let $T_{\mathrm{cpt}}$ be a compact torus such that

$$
T_{\mathrm{cpt}} \mathcal{A}\left(\operatorname{Ad}_{G}(\Gamma)\right)=\mathcal{A}\left(\operatorname{Ad}_{G}(G)\right)
$$


Then there exists a subgroup $\tilde{\Gamma}$ of finite index in $\Gamma$ and a simply connected normal subgroup $\tilde{G}$ of $T_{\mathrm{cpt}} \ltimes G$ such that

$$
\mathcal{A}\left(\operatorname{Ad}_{\tilde{G}}(\tilde{\Gamma})\right)=\mathcal{A}\left(\operatorname{Ad}_{\tilde{G}}(\tilde{G})\right) .
$$

Therefore, $\tilde{G} / \tilde{\Gamma}$ is diffeomorphic to $G / \tilde{\Gamma}$ and $H_{\mathrm{dR}}^{*}(G / \tilde{\Gamma}) \cong H^{*}(\tilde{\mathfrak{g}})$, where $\tilde{\mathfrak{g}}$ is the Lie algebra of $\tilde{G}$.

Observe that $H_{\mathrm{dR}}^{*}(G / \Gamma) \cong H_{\mathrm{dR}}^{*}(G / \tilde{\Gamma})^{\Gamma / \tilde{\Gamma}}$ (the invariants by the action of the finite group $\Gamma / \tilde{\Gamma})$.

In Section 6 we give a proof of the previous theorem and we apply it to the special class of almost Abelian Lie groups. We recall that a simply connected Lie group $G$ is called almost Abelian if its Lie algebra $\mathfrak{g}$ has an Abelian ideal of codimension 1. In [13] the Mostow condition was studied for simply connected almost Abelian Lie groups and the first Betti number of a compact quotient of a simply connected almost Abelian Lie group by a lattice was already determined in [5].

Acknowledgements. We would like to thank Diego Conti and Daniel Guan for useful comments. We also would like to thank the referee for valuable remarks which improved the contents of the paper.

\section{General properties of lattices in solvable Lie groups}

Let $G$ be a real Lie group. We recall that an element $g \in G$ is unipotent (Adunipotent) if the operator $\operatorname{Ad}_{g}$ on the Lie algebra $\mathfrak{g}$ of $G$ is unipotent, i.e. all of its eigenvalues are equal to 1 or equivalently there exists a positive integer $k$ such that $\left(\operatorname{Ad}_{g}-i d\right)^{k}=0$. A subgroup $H \subset G$ is unipotent if all its elements are unipotent. An element $g \in G$ is called semisimple (Ad-semisimple) if the operator $\operatorname{Ad}_{g}$ on the Lie algebra $\mathfrak{g}$ of $G$ is $\mathbb{C}$-diagonalizable.

Given two subgroups $H$ and $H^{\prime}$ of $G$, the commutator $\left[H, H^{\prime}\right]$ is the subgroup of $G$ generated by the elements

$$
\{h, k\}=h k h^{-1} k^{-1}, \quad h \in H, k \in H^{\prime} .
$$

Given a Lie group $G$ one may consider the descending chain of normal subgroups

$$
G_{0}=G \supset G_{1}=[G, G] \supset \cdots \supset G_{i+1}=\left[G_{i}, G\right] \supset \ldots .
$$

The Lie group $G$ is nilpotent if the previous descending chain degenerates, i.e. if $G_{i}=\{e\}$, where $e$ is the identity element, for all $i$ greater than or equal to some $k$. 
One may consider another descending chain of normal subgroups, called the derived series

$$
G_{(0)}=G \supset G_{(1)}=[G, G] \supset \cdots \supset G_{(i+1)}=\left[G_{(i)}, G_{(i)}\right] \supset \ldots .
$$

and $G$ is solvable if the previous chain degenerates. A solvable Lie group is called completely solvable if every eigenvalue $\lambda$ of every operator $\operatorname{Ad}_{g}, g \in G$, is real.

The unipotent radical $\operatorname{Rad}_{u} G$ of a Lie group $G$ is its largest normal subgroup consisting of unipotent elements. In particular $\operatorname{Rad}_{u} G$ is a nilpotent subgroup of $G$. We recall that the nilradical $N$ of a Lie group $G$ is its largest connected nilpotent normal subgroup. In the general case $\operatorname{Rad}_{u} G \subset N$. The nilradical $N$ is unique and the quotient $G / N$ is a simply-connected Abelian Lie group. Thus $G$ is an extension of $N$ by an Abelian Lie group $\mathbb{R}^{k}$, i.e. $G$ satisfies the exact sequence

$$
1 \rightarrow N \rightarrow G \rightarrow \mathbb{R}^{k} \rightarrow 1,
$$

for some $k \geq 0$. If $G$ is solvable, the commutator $[G, G]$ is nilpotent and therefore the commutator $[G, G]$ is a subgroup of $\operatorname{Rad}_{u} G$ and thus it is a subgroup of $N$. Any compact solvable Lie group is commutative and hence it is a torus. Any simply connected solvable Lie group is homeomorphic to a vector space, although the exponential map exp : $\mathfrak{g} \rightarrow G$ is not necessarily injective or surjective. Moreover, a connected solvable Lie group $G$ is simply connected if and only if $G$ has no non trivial compact subgroups.

A discrete subgroup $\Gamma$ of a solvable Lie group $G$ is a lattice if $G$ / $\Gamma$ has a finite invariant measure. By [30, Theorem 3.1] if $G$ is a solvable Lie group with countably many connected components and $H$ is a closed subgroup, then $G / H$ has a $G$-invariant finite measure if and only if $G / H$ is compact. Therefore, a uniform discrete subgroup of a connected solvable Lie group $G$ is always a lattice. The identity component $\Gamma^{0}$ of a lattice $\Gamma$ of $G$ belongs to the nilradical $N$ of $G$ and the space $N / N \cap \Gamma$ is compact.

For any lattice $\Gamma$ of $G$ we have that $\Gamma \cap N$ is a lattice in the nilradical $N$ $[1,25,30]$. In general if $G$ is a connected Lie group and $G / \Gamma$ is a compact quotient of $G$ by a uniform discrete subgroup, then the normalizer $N_{G}\left(\Gamma^{0}\right)$ is uniform in $G$ ( $\Gamma^{0}$ denotes the connected component of the identity). In the case of solvable Lie groups there is no simple criterion for the existence of a lattice in a connected and simply connected solvable Lie group. The only general necessary condition is that the Lie group $G$ has to be unimodular (see [22, Lemma 6.2]), i.e. the trace $\operatorname{tr}\left(\operatorname{ad}_{X}\right)$ vanishes for any $X$ in the Lie algebra $\mathfrak{g}$ of $G$. Since the Lie algebra $\mathfrak{g}$ is unimodular, Poincaré duality holds for the (co)-homology of $\mathfrak{g}$ (see for instance [14, Section 5.11]).

\section{Linear algebraic groups}

We recall that a subgroup $A$ of $\operatorname{SL}(n, \mathbb{R})$ is a real algebraic group or Zariski closed if $A$ is the set of zeros of a polynomial in matrix entries with real coefficients. 
One can also define a real algebraic group as a subgroup of $\operatorname{GL}(n, \mathbb{R})$ using the embedding $\rho: \operatorname{GL}(n, \mathbb{R}) \rightarrow \operatorname{SL}(n+1, \mathbb{R})$ defined by

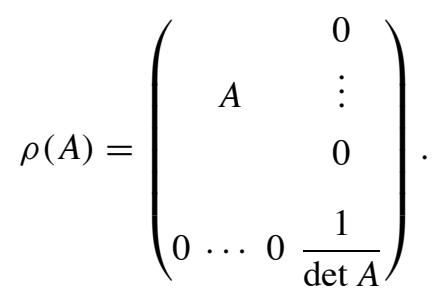

So, a subgroup $A$ of $\operatorname{GL}(n, \mathbb{R})$ is a real algebraic group if it is the set of zeros of some collection of regular functions on $\operatorname{GL}(n, \mathbb{R})$, where for regular functions we mean real valued functions on $\operatorname{GL}(n, \mathbb{R})$ for which there is a polynomial $p$ in $n^{2}+1$ variables, such that $f(g)=p\left(g_{i j}, \operatorname{det}\left(g^{-1}\right)\right)$. As a subset of $\mathbb{R}^{n^{2}}$, a real algebraic group carries both the natural Euclidean topology which turns it into a real Lie group and also the Zariski topology induced from $\operatorname{GL}(n, \mathbb{R})$.

A subgroup $A$ of $\operatorname{GL}(n, \mathbb{R})$ is an almost algebraic group or almost Zariski closed if there is a real algebraic group $B \subset \operatorname{GL}(n, \mathbb{R})$ such that $B^{0} \subset A \subset B$, where $B^{0}$ is the identity component of $B$ in the Euclidean topology. The difference between being real algebraic and almost real algebraic is slight, since $B^{0}$ has always finite index in $B$.

A real algebraic group $U$ is unipotent if every element $g$ of $U$ is unipotent, i.e., there exists a positive integer $k$ such that $(g-I)^{k}=0$. A real algebraic group $T$ is a torus if $T$ is Abelian, Zariski connected and every element $g$ of $T$ is semisimple, i.e. $g$ is $\mathbb{C}$-diagonalizable. A torus $T$ is $\mathbb{R}$-split if every element of $T$ is diagonalizable over $\mathbb{R}$.

If $G$ is a Zariski-connected, solvable real algebraic group, then ( $c f$. [6]):

(1) the set $U$ of all unipotent elements of $G$ is a normal subgroup, which is called the unipotent radical of $G$;

(2) $G / U$ is Abelian;

(3) for every maximal torus $T$ of $G$, one has $G=T \ltimes U$.

In particular, a Zariski-connected, solvable real algebraic group $G$ has a decomposition

$$
G=\left(T_{\text {split }} \cdot T_{\text {cpt }}\right) \ltimes U,
$$

where $T_{\text {split }}$ is the maximal $\mathbb{R}$-split subtorus of $T, T_{\text {cpt }}$ is the maximal compact subtorus of $T$ and $T_{\text {split }} \cap T_{\text {cpt }}$ is finite. In any connected almost algebraic group $G$ all the maximal compact tori are conjugate.

The (almost) Zariski closure $\mathcal{A}(H)$ of a subgroup $H$ of an (almost) algebraic group is the unique smallest (almost) algebraic subgroup that contains $H$. If $K$ is the Zariski closure of $H$, then $\mathcal{A}(H)=K^{0} H$, i.e., $\mathcal{A}(H)$ is the union of all connected components of $K$ that intersect $H$. For instance, if $H$ is connected, then $\mathcal{A}(H)=K^{0}$, so the almost Zariski closure of a connected group is connected. 
In general, given a Lie group $G$, we recall that $\operatorname{Ad}_{G}(G)$ is the subgroup of $\mathrm{GL}(\mathfrak{g})$ generated by $e^{\operatorname{ad}_{X}}$, for all $X \in \mathfrak{g}$. Since Ad $\exp X=e^{\operatorname{ad}_{X}}$, we have that $\operatorname{Ad}_{G}(G)$ has $\operatorname{ad}(\mathfrak{g})$ as Lie algebra. Moreover $\operatorname{Ad}_{G} G$ is isomorphic to $G / C$, where $C$ is the center of $G$. It turns out that if $G$ is a simply connected solvable Lie group then $\operatorname{Ad}_{G}(G)$ is a solvable algebraic group. If $H$ is a subgroup of a connected Lie group $G$, we will denote by $\mathcal{A}\left(\operatorname{Ad}_{G}(H)\right)$ the (almost) Zariski closure of $\operatorname{Ad}_{G}(H)$ in the real algebraic group $\operatorname{Aut}(\mathfrak{g})$, where $\mathfrak{g}$ is the Lie algebra of $G$. Since $G$ is connected, by [35, Proposition 3.8] $\mathcal{A}\left(\operatorname{Ad}_{G}(G)\right)$ acts on $G$ and centralizes the center of $G$. If $G$ is in addition simply connected, $\operatorname{Aut}(G) \cong \operatorname{Aut}(\mathfrak{g})$ so $\mathcal{A}\left(\operatorname{Ad}_{G}(G)\right)$ lifts to a group of automorphisms of $G$.

The set of unipotent elements in $\mathcal{A}\left(\operatorname{Ad}_{G}(G)\right)$ forms a normal subgroup $U$, the unipotent radical, and if $T$ is any maximal reductive subgroup of $\mathcal{A}\left(\operatorname{Ad}_{G}(G)\right)(\operatorname{a} \max -$ imal algebraic torus), we have a semidirect product decomposition $\mathcal{A}\left(\operatorname{Ad}_{G}(G)\right)=$ $T \ltimes U$. If $\Gamma$ is a lattice of $G$, one has that $\mathcal{A}\left(\operatorname{Ad}_{G}(\Gamma)\right)$ contains $U(c f .[30$, p. 45]) and so $\mathcal{A}\left(\operatorname{Ad}_{G}(\Gamma)\right)$ can be written as $S \ltimes U$ where $S=\mathcal{A}\left(\operatorname{Ad}_{G}(\Gamma)\right) \cap T$. Note that $S$ leaves the Lie algebra $\mathfrak{n}$ of the nilradical of $G$ invariant as well as the Lie algebra of the identity connected component of $\Gamma$.

Theorem 3.1 (Borel density theorem [35, Corollary 3.29]). Let $\Gamma$ be a lattice of a simply connected solvable Lie group $G$, then there exists a maximal compact torus $T_{\text {cpt }} \subset \mathcal{A}\left(\operatorname{Ad}_{G}(G)\right)$, such that

$$
\mathcal{A}\left(\operatorname{Ad}_{G}(G)\right)=T_{\mathrm{cpt}} \mathcal{A}\left(\operatorname{Ad}_{G}(\Gamma)\right) .
$$

Since for a completely solvable Lie group $G$ the adjoint representation has only real eigenvalues, as a consequence (see for instance [35]), we have the following:

Proposition 3.2. Let $\Gamma$ be a lattice of a simply connected completely solvable Lie group $G$, then $\mathcal{A}\left(\operatorname{Ad}_{G}(G)\right)$ has no non trivial connected compact subgroups and thus as a consequence $\mathcal{A}\left(\operatorname{Ad}_{G}(G)\right)=\mathcal{A}\left(\operatorname{Ad}_{G}(\Gamma)\right)$.

In particular this is the case when $G$ is nilpotent, since in this case $\operatorname{Ad}_{G}(G)$ is unipotent and thus $\mathcal{A}\left(\operatorname{Ad}_{G}(G)\right)$ coincides with $\operatorname{Ad}_{G}(G)$. Note that when $G$ is a simply connected nilpotent Lie group, by the Birkhoff embedding theorem [1] there exists a monomorphism $B: G \rightarrow B(n, \mathbb{R})$, where $B(n, \mathbb{R}) \subset \operatorname{SL}(n, \mathbb{R})$ denotes the group of strictly upper triangular matrices of order $G$ (with all diagonal elements equal to 1), which allows to treat $G$ as an algebraic group all of whose connected subgroups are algebraic. In the special case of a solvable Lie group $G$ for which all the eigenvalues of $\operatorname{Ad}(g), g \in G$, belong to the the unit circle, then the algebraic torus $T$ in $\mathcal{A}\left(\operatorname{Ad}_{G}(G)\right)=T \ltimes U$ is compact.

\section{4. de Rham Cohomology of solvmanifolds}

An important result related to lattices for compact solvmanifolds is the following:

Theorem 4.1 ([30, Theorem 3.6]). If $\Gamma_{1}$ and $\Gamma_{2}$ are lattices in simply connected solvable Lie groups $G_{1}$ and $G_{2}$, respectively, and $\Gamma_{1} \cong \Gamma_{2}$, then $G_{1} / \Gamma_{1}$ is diffeomorphic to $G_{2} / \Gamma_{2}$. 
As a consequence two compact solvmanifolds with isomorphic fundamental groups are diffeomorphic.

Example 4.2. Let $G$ be the semidirect product $\mathbb{R} \ltimes_{\varphi} \mathbb{R}^{2}$, where

$$
\varphi(t)=\left(\begin{array}{cc}
\cos (2 \pi t) & \sin (2 \pi t) \\
-\sin (2 \pi t) & \cos (2 \pi t)
\end{array}\right), \quad t \in \mathbb{R} .
$$

The element $1 \in \mathbb{R}$ and the standard integer lattice $\mathbb{Z}^{2}$ generate a lattice $\Gamma$ isomorphic to $\mathbb{Z}^{3}$. Applying the previous theorem one has that $G / \Gamma$ is diffeomorphic to the torus $T^{3}$.

In general $\operatorname{Ad}_{G}(\Gamma)$ and $\operatorname{Ad}_{G}(G)$ do not have the same Zariski closures. A simple example for which $\mathcal{A}\left(\operatorname{Ad}_{G}(G)\right) \neq \mathcal{A}\left(\operatorname{Ad}_{G}(\Gamma)\right)$ is the following.

Example 4.3. For the previous Example 4.2 we have by [25] that $\operatorname{Ad}_{G}(\Gamma)$ is Abelian and then $\mathcal{A}\left(\operatorname{Ad}_{G}(\Gamma)\right)$ is also Abelian. $\operatorname{But}^{A_{d}}(G)$ is not Abelian. Indeed, $\mathcal{A}\left(\operatorname{Ad}_{G}(G)\right)$ is locally isomorphic to $G$. Then in this case $\mathcal{A}\left(\operatorname{Ad}_{G}(\Gamma)\right) \neq \mathcal{A}\left(\operatorname{Ad}_{G}(G)\right)$.

Let $H^{*}(\mathfrak{g})$ be the Chevalley-Eilenberg cohomology of the Lie algebra $\mathfrak{g}$ of $G$. In general, there is a natural injective map $H^{*}(\mathfrak{g}) \rightarrow H_{\mathrm{dR}}^{*}(M)$ which is an isomorphism in the following cases:

Theorem 4.4. Let $G / \Gamma$ be a compact solvmanifold.

(1) [19] If $G$ is completely solvable, then $H_{\mathrm{dR}}^{*}(G / \Gamma) \cong H^{*}(\mathfrak{g})$.

(2) [30, Corollary 7.29] If $\operatorname{Ad}_{G}(\Gamma)$ and $\operatorname{Ad}_{G}(G)$ have the same Zariski (i.e. real algebraic) closure in $\mathrm{GL}(\mathfrak{g})$, then $H_{\mathrm{dR}}^{*}(G / \Gamma) \cong H^{*}(\mathfrak{g})$.

For the sake of completness we will include the proof of (2), generalizing that given by Nomizu [27] in the case of a nilmanifold.

Proof of (2). Let $G_{(k)}=\left[G_{(k-1)}, G_{(k-1)}\right]$ and $\Gamma_{(k)}=\left[\Gamma_{(k-1)}, \Gamma_{(k-1)}\right]$ be the derived series for $G$ and $\Gamma$. Remark that $G_{(k)}$ is nilpotent for any $k \geq 1$ and that since $\mathcal{A}\left(\operatorname{Ad}_{G}(G)\right)=\mathcal{A}\left(\operatorname{Ad}_{G}(\Gamma)\right)$ a general result on lattices in nilpotent Lie groups ( [ $[30$, Theorem 2.1]) implies that $G_{(k)} / \Gamma_{(k)}$ is compact for any $k$. Hence $G_{(k)} / \Gamma \cap G_{(k)}$ is compact for any $k$.

Let $r$ be the last non-zero term in the derived series of $G$. Namely $G_{(r+1)}=(e)$ and $G_{(r)}=: A \neq(e)$. Observe that $A$ is Abelian. So $A / A \cap \Gamma:=T^{m}$ is a compact torus. Thus, $\bar{M}:=G / A \Gamma$ is a compact solvmanifold with dimension smaller than $M:=G / \Gamma$ and $T^{m} \hookrightarrow M \stackrel{\pi}{\rightarrow} \bar{M}$ is a fibration.

Next, consider the Leray-Serre spectral sequence $E_{*}^{p, q}$ associated with the above fibration. One has

$$
\begin{aligned}
& E_{2}^{p, q}=H_{\mathrm{dR}}^{p}\left(\bar{M}, H_{\mathrm{dR}}^{q}\left(T^{m}\right)\right) \cong H_{\mathrm{dR}}^{p}(\bar{M}) \otimes \bigwedge^{q} \mathbb{R}^{m}, \\
& E_{\infty}^{p, q} \Rightarrow H_{\mathrm{dR}}^{p+q}(M) .
\end{aligned}
$$


We now define a second spectral sequence $\tilde{E}_{*}^{p, q}$ which is the Leray-Serre spectral sequence relative to the complex of $G$-invariant forms (i.e., the Chevalley-Eilenberg complex). Since $\bigwedge^{*} \mathfrak{g}^{*}$ is subcomplex of $\bigwedge^{*} M, \tilde{E}_{*}^{p, q} \subseteq E_{*}^{p, q}$ and

$$
\tilde{E}_{2}^{p, q}=H^{p}(\mathfrak{g} / \mathfrak{a}) \otimes \bigwedge^{q} \mathbb{R}^{m}, \quad \tilde{E}_{\infty}^{p, q} \Rightarrow H^{p+q}(\mathfrak{g}),
$$

where $\mathfrak{a}$ denotes the Lie algebra of $A$.

Now, since $\bar{M}$ is a compact solvmanifold of lower dimension than $M$, an inductive argument on the dimension shows that $H_{\mathrm{dR}}^{p}(\bar{M}) \cong H^{p}(\mathfrak{g} / \mathfrak{a})$ for any $p$. Thus $E_{2}=\tilde{E}_{2}$ and one has the equality $E_{\infty}=\tilde{E}_{\infty}$. Therefore, $H_{\mathrm{dR}}^{k}(M)=$ $H_{\mathrm{dR}}^{k}(G / \Gamma) \cong H^{k}(\mathfrak{g})$ for any $k$.

Remark 4.5. By Proposition 3.2 it turns out that (1) is then a particular case of (2). Moreover, one can have the isomorphism $H^{*}(\mathfrak{g}) \cong H_{\mathrm{dR}}^{*}(G / \Gamma)$ even if $\operatorname{Ad}_{G}(\Gamma)$ and $\operatorname{Ad}_{G}(G)$ do not have the same algebraic closure (see Example 6.5).

For a solvable Lie algebra $\mathfrak{g}$ one has always that $[\mathfrak{g}, \mathfrak{g}] \neq \mathfrak{g}$. Thus, as a consequence, the first Betti number $b_{1}(G / \Gamma)$ of a compact solvmanifold $G / \Gamma$ satisfies the inequality:

$$
b_{1}(G / \Gamma) \geq 1 .
$$

(see also [5, Corollary 3.12]). In the particular case of a nilmanifold $G / \Gamma$, one has $b_{1}(G / \Gamma) \geq 2$.

\section{Semisimple splitting and nilshadow}

Let $G$ be a connected and simply-connected solvable Lie group. We recall that $G$ can be viewed as the extension of the nilradical $N$ by an Abelian Lie group $\mathbb{R}^{k}$, but in general this extension does not split. If $G$ is a solvable algebraic real group, then $G=A \ltimes U$, where $U$ is a unipotent radical and $A$ is an Abelian subgroup, consisting of semisimple elements [21]. Thus in this case $G$ splits.

Definition 5.1. A solvable simply connected Lie group $G$ is splittable if $G$ can be represented in the form of a semidirect product $G=A \ltimes_{\varphi} N$, where $N$ is the nilradical of $G, A$ is an Abelian subgroup and the subgroup $\varphi(A) \subset \operatorname{Aut}(N)$ consists of semisimple automorphisms.

By definition $\operatorname{ker} \varphi$ is a discrete subgroup of $A$. Malcev introduced for an arbitrary simply connected Lie group the general notion of splitting.

Definition 5.2. A semisimple splitting or a Malcev splitting of a simply connected solvable Lie group $G$ is an imbedding $\iota: G \rightarrow M(G)$ of the group $G$ in a splittable simply-connected solvable Lie group $M(G)$, such that if $M(G)=T_{G} \ltimes U_{G}$, where $U_{G}$ is the nilradical of $M(G)$ and $T_{G}$ is an Abelian subgroup acting on $U_{G}$ by semisimple automorphisms, then

(1) $M(G)=\iota(G) U_{G}$ (product of subgroups);

(2) $M(G)=T_{G} \ltimes \iota(G)$ (semidirect product of $T_{G}$ with the normal subgroup $\iota(G)$ ). 
Gorbatsevich proved in [11] that, for any simply connected solvable Lie group, a Malcev splitting exists and it is unique. Then the general idea for semisimple splitting is to embed solvable and simply connected Lie groups in splits groups. We briefly recall the construction. Let $G$ be a simply connected solvable Lie group and Ad $: G \rightarrow \operatorname{Aut}(\mathfrak{g})$ the adjoint representation of $G$ in the automorphism group $\operatorname{Aut}(\mathfrak{g})$ of the Lie algebra $\mathfrak{g}$ of $G$. The real algebraic group $\operatorname{Aut}(\mathfrak{g})$ splits as follows:

$$
\operatorname{Aut}(\mathfrak{g})=(P T) \ltimes U,
$$

where $P$ is a maximal semisimple subgroup, $T$ is an Abelian subgroup of semisimple automorphisms of $\mathfrak{g}$ that commute with $P$ (and the intersection $P \cap T$ is discrete) and $U$ is the unipotent radical of $\operatorname{Aut}(\mathfrak{g})$.

The group $\operatorname{Ad}_{G}(G)$ is a connected solvable normal subgroup of $\operatorname{Aut}(\mathfrak{g})$ and thus it is contained in the radical $T \ltimes U$ of the algebraic group $\operatorname{Aut}(\mathfrak{g})$. Let $\theta: T \ltimes$ $U \rightarrow T$ be the epimorphism induced by projection and $T_{G}=\theta\left(\operatorname{Ad}_{G}(G)\right)$. Thus $T_{G}$ is a connected Abelian group of semisimple automorphisms of $\mathfrak{g}$ and therefore of $G$ itself. The connected solvable Lie group $M(G)=T_{G} \ltimes G$ is precisely the semisimple splitting of $G$. It is possible to show that (see [2]) if $U_{G}$ is the nilradical of $M(G)$, then $M(G)$ is the semidirect product $M(G)=T_{G} \ltimes_{v_{s}} U_{G}$. Moreover, $M(G)=G U_{G}$, i.e. $G$ and $U_{G}$ generate $M(G)$. The new Lie group $M(G)$ is splittable, since the exact sequence

$$
1 \rightarrow U_{G} \rightarrow M(G) \rightarrow \mathbb{R}^{k} \rightarrow 1
$$

splits. The nilradical $U_{G}$ is also called the nilshadow of $G$. If we denote by

$$
p: M(G)=T_{G} \ltimes U_{G} \rightarrow T_{G}, \quad \pi: M(G)=T_{G} \ltimes U_{G} \rightarrow U_{G},
$$

the projections over $T_{G}$ and $U_{G}$, then the restriction $p: G \rightarrow U_{G}$ is a group epimorphism and the restriction $\pi: G \rightarrow U_{G}$ is a diffeomorphism. Moreover,

$$
\left[U_{G}, U_{G}\right] \subset N, \quad\left[T_{G}, U_{G}\right] \subset N
$$

(see for instance [32]). Following [5] we give an explicit description of $U_{G}$ and of the action $v_{s}$ of $T_{G}$ on $U_{G}$. More precisely, if $N$ is the nilradical of $G$, we have that there exists a maximal connected and simply connected nilpotent Lie subgroup $K$ of $G$ such that $G$ is the product $K \cdot N$ [10, Proposition 3.3]. The nilpotent Lie group $K$ is also called a supplement for $N$ in $G$ and it is unique up to conjugation.

There is a natural action of $K$ on $N$ induced by conjugation in $G$. Consider the action

$$
\tilde{\phi}: K \longrightarrow \text { Aut }(G)
$$

given by

$$
\tilde{\phi}(a)(k \cdot n)=k \cdot\left(\left.I_{a}\right|_{N}\right)_{S}(n),
$$


where $\left(\left.I_{a}\right|_{N}\right)_{S}(n)$ is the semisimple part of the inner automorphism of $N$, obtained by conjugating the elements of $N$ by $a$. Then, there is an induced action $\phi$ of

$$
T_{G}=K /(N \cap K) \cong(K N) / N \cong G / N \cong \mathbb{R}^{k} .
$$

on $G$ which makes the following diagram commutative:

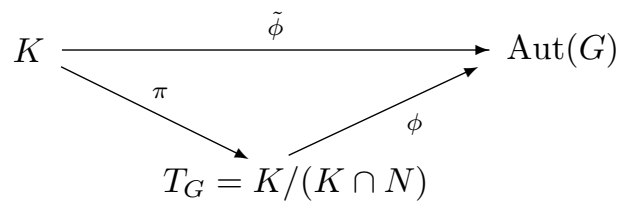

The semisimple splitting $M(G)$ coincides with the semidirect product

$$
T_{G} \ltimes_{\phi} G .
$$

Indeed, one has an imbedding $\iota: G \longrightarrow T_{G} \ltimes_{\phi} G$ of $G$ into $T_{G} \ltimes_{\phi} G$ such that $\iota(G)$ is a closed normal subgroup of $T_{G} \ltimes_{\phi} G$. Moreover, by [5] the nilradical of $T_{G} \ltimes_{\phi} G$ is:

$$
U_{G}=\left\{\pi\left(k^{-1}\right) \cdot k \mid k \in K\right\} \cdot N=\left\{\left(\pi\left(k^{-1}\right), k \cdot n\right) \mid k \in K, n \in N\right\} \subset T_{G} \ltimes_{\phi} G
$$

and $T_{G} \cap U_{G}=\{e\}$. Then

$$
T_{G} \ltimes_{\phi} G=T_{G} \ltimes_{v_{s}} U_{G},
$$

coincides with the semisimple splitting $M(G)$ of $G$, where $U_{G}$ is the nilradical of $M(G)$ and $v_{s}$ is the semisimple automorphism:

$$
v_{s}(t)\left(\pi\left(k^{-1}\right) \cdot(k \cdot n)\right):=t \cdot \pi\left(k^{-1}\right) \cdot(k \cdot n) \cdot t^{-1}=\pi\left(k^{-1}\right) \cdot\left(k \cdot \tilde{\phi}\left(k_{t}\right)(n)\right),
$$

for any $t \in T_{G}, k \in K, n \in N$ and any $k_{t} \in \pi^{-1}(\{t\})$. In this way, one has the diagonal embedding:

$$
\Delta: G=K \cdot N \rightarrow M(G)=\mathbb{R}^{k} \ltimes G, k \cdot n \mapsto\left(\pi\left(k^{-1}\right), k \cdot n\right)
$$

and the nilradical $U_{G}$ coincides with the image $\Delta(G)$. By construction, $U_{G}$ is diffeomorphic to $G$ but it has a different product that makes it nilpotent.

More explicitly, at the level of Lie algebras we have that there exists a vector space $V \cong \mathbb{R}^{k}$ such that $\mathfrak{g}=V \oplus \mathfrak{n}$ and $\operatorname{ad}(A)_{S}(B)=0$, for any $A, B \in V$, where $\operatorname{ad}(A)_{s}$ is the semisimple part of $\operatorname{ad}(A)$. By considering $V \cong \mathbb{R}^{k}$ as an Abelian Lie algebra, then the Lie algebra of the semisimple splitting $M(G)$ of $G$ is $V \ltimes_{\text {ad( }(\ldots)_{s}} \mathfrak{g}$, i.e.

$$
[(A, X),(B, Y)]=\left(0,[X, Y]+\operatorname{ad}(A)_{s}(Y)-\operatorname{ad}(B)_{s}(X)\right),
$$

with nilradical (the Lie algebra of $U_{G}$ )

$$
\mathfrak{u}_{G}=\left\{\left(-X_{V}, X\right), \mid X \in \mathfrak{g}\right\},
$$

where $X_{V}$ denotes the component of $X$ in $V$. Note that $\mathfrak{u}_{G}$ is isomorphic to $\mathfrak{g}$ only as a vector space. 
Example 5.3. If $G$ is nilpotent, then by definition $M(G)=G$. Now suppose in addition that $G$ is splittable, i.e. $G=A \ltimes N$, where $N$ is the nilradical of $G$ and the Abelian group $A$ acts on $N$ by semisimple automorphisms. Let $*: A \rightarrow \operatorname{Aut}(N)$ be the natural homomorphism, then the image $*(A)=A^{*}$ is an Abelian subgroup of $\operatorname{Aut}(N)$ consisting of semisimple elements and $A^{*}$ can be considered as a subgroup of Aut.

Therefore $T_{G}=A^{*}, M(G)=A^{*} \ltimes G$ and the nilshadow $U_{G}$ is

$$
\left\{\left(\left(a^{*}\right)^{-1}, a\right) \mid a \in A\right\} \cdot N .
$$

In this case one says that the nilshadow is the image of the "anti-diagonal" map

$$
A \ltimes N \rightarrow A^{*} \ltimes(A \ltimes N),(a, n) \mapsto\left(\left(a^{*}\right)^{-1},(a, n)\right) .
$$

By construction there is a strict relation between the semisimple splitting of $G$ and the algebraic closure $\mathcal{A}\left(\operatorname{Ad}_{G}(G)\right)$ of $\operatorname{Ad}_{G}(G)$. Since $G$ is solvable and simply connected, then $\operatorname{Aut}(G) \cong \operatorname{Aut}(\mathfrak{g}), \operatorname{Ad}_{G}(G)$ is a solvable algebraic group and it is connected as algebraic group. Therefore its closure $\mathcal{A}\left(\operatorname{Ad}_{G}(G)\right)$, in $\operatorname{GL}(\mathfrak{g})$, has the Chevalley decomposition

$$
\mathcal{A}\left(\operatorname{Ad}_{G}(G)\right)=T \ltimes U
$$

where $U$ is the unipotent radical (i.e. the largest normal subgroup of $\mathcal{A}\left(\operatorname{Ad}_{G}(G)\right)$ consisting of unipotent elements) and $T$ is an Abelian subgroup (a maximal algebraic torus) acting by semisimple (i.e. completely reducible) linear operators. Although $U$ is nilpotent, it is important to note that $U$ need not to be the nilradical of $\mathcal{A}\left(\operatorname{Ad}_{G}(G)\right)$. Note that $T$ is any maximal reductive subgroup of $\mathcal{A}\left(\operatorname{Ad}_{G}(G)\right)$ and it is a standard fact that all such groups $T$ are conjugate by elements of $U$.

The group $\operatorname{Aut}(\mathfrak{g})$ is algebraic and $\operatorname{Ad}_{G}(G) \subset \operatorname{Aut}(\mathfrak{g})$. Therefore, the group $T$ may be considered as a subgroup of $\operatorname{Aut}(\mathfrak{g}) \cong \operatorname{Aut}(G)$. There is a natural epimorphism with kernel $U$ :

$$
\theta: T \ltimes U \longrightarrow T \text {. }
$$

Let $T_{G}^{*}=\theta\left(\operatorname{Ad}_{G}(G)\right)$ be the image of $\operatorname{Ad}_{G}(G)$ by the previous epimorphism, i.e. $T_{G}^{*}$ can be viewed as the image of $\operatorname{Ad}_{G}(G)$ in $T$.

We can view either $T$ or $T_{G}^{*}$ as a group of automorphisms of $G$. By using the imbeddings

$$
T_{G}^{*} \longrightarrow \operatorname{Aut}(G), \quad T \longrightarrow \operatorname{Aut}(G)
$$

we may construct the two semi-direct products

$$
T_{G}^{*} \ltimes G \quad \text { and } \quad T \ltimes G .
$$

The semisimple splitting is the universal covering $M(G)=T_{G} \ltimes G=\mathbb{R}^{k} \ltimes G$ of the Lie group $T_{G}^{*} \ltimes G$. In general $T_{G}$ is not an algebraic subgroup of $\operatorname{Aut}(\mathfrak{g})$ and it could possibly be not closed in the Euclidean topology, but if $G$ admits lattices, then the subgroup $T_{G}$ is closed in the Euclidean topology. 
Remark 5.4. The semidirect product $T \ltimes G$ splits as a semi-direct product $T \ltimes U_{G}$, since the nilradical $U_{G}$ of $M(G)$ coincides with the nilradical of $T \ltimes G$ and $T \ltimes G$ admits the structure of algebraic group (defined over the real numbers). Moreover, $G$, as a subgroup of $T \ltimes G$, is Zariski dense and hence one may think of $T \ltimes G$ as a kind of "algebraic hull" of $G$.

\section{The nilshadow map and its applications to the cohomology}

Let $G$ be a connected, solvable real algebraic group, then $G$ is a semidirect product of the form $G=T \ltimes U$, where $T=T_{\text {split }} \times T_{\text {cpt }}$ is a torus and $U$ is nilpotent. One can see, following the paper [35], that the subgroup $T$ acts on $G$ (by conjugation), thus one can construct the semidirect product:

$$
T \ltimes G=T \ltimes(T \ltimes U),
$$

and the nilshadow $\Delta(G)$ can be embedded in $T \ltimes G$ by the map:

$$
(t, u) \rightarrow\left(t^{-1}, t, u\right) \in T \ltimes(T \ltimes U),
$$

where $t^{-1}$ acts on $G=T \ltimes U$ by conjugation.

The anti-diagonal imbedding of $T$ sends it into the center of $T \ltimes G$. In this way the nilshadow $U_{G}$ can be viewed as the image of the map:

$$
\Delta: G \rightarrow T \ltimes G, g \mapsto \Delta(g)=\left(\pi(g)^{-1}, g\right),
$$

where $\pi: G \rightarrow T$ is the projection, i.e. $U_{G}=\Delta(G)$. This antidiagonal embedding of $T$ is the main idea of the nilshadow construction.

For the general case of a solvable (non nilpotent) Lie group $G$, which may not be real algebraic, one has that $\mathcal{A}\left(\operatorname{Ad}_{G}(G)\right)=T \ltimes U$ is not unipotent, i.e. a maximal torus $T=T_{\text {split }} \times T_{\text {cpt }}$ of $\mathcal{A}\left(\operatorname{Ad}_{G}(G)\right)$ is non trivial. The basic idea for the construction of the nilshadow is to kill $T$ in order to obtain a nilpotent group. In order to do this one can define, following [35], a natural homomorphism $\pi: G \rightarrow T$, which is the composition of the homomorphisms:

$$
\pi: G \stackrel{\mathrm{Ad}}{\longrightarrow} \rightarrow \mathcal{A}\left(\operatorname{Ad}_{G}(G)\right) \stackrel{\text { projection }}{\longrightarrow} T,
$$

and the map

$$
\Delta: G \rightarrow T \ltimes G, g \mapsto \Delta(g)=\left(\pi(g)^{-1}, g\right) .
$$

The traditional nilshadow construction kills the entire maximal torus $T$ in order to get the nilpotent Lie group $\Delta(G)$. Witte introduced in [35] a variation, killing only a subtorus $S$ of $T$. It is well known that, for every subtorus $S$ of a compact torus $T$, there is a torus $S^{\perp}$ complementary to $S$ in $T$, i.e. such that $T=S \times S^{\perp}$.

As a consequence of [35, Proposition 8.2] now we are able to prove the main theorem.

Proof of the main theorem. By [30, Theorem 6.11, p. 93] it is not restrictive to suppose that $\mathcal{A}\left(\operatorname{Ad}_{G}(\Gamma)\right)$ is connected. Otherwise we pass from $\Gamma$ to a finite index subgroup $\tilde{\Gamma}$. This is equivalent to passing from $M=G / \Gamma$ to the space $G / \tilde{\Gamma}$ 
which is a finite-sheeted covering of $M$. Let $T_{\text {cpt }}$ be a maximal compact torus of $\mathcal{A}\left(\operatorname{Ad}_{G} G\right)$ which contains a maximal compact torus $S_{\text {cpt }}^{\perp}$ of $\mathcal{A}\left(\operatorname{Ad}_{G}(\tilde{\Gamma})\right)$. There is a natural projection from $\mathcal{A}\left(\operatorname{Ad}_{G}(G)\right)$ to $T_{\text {cpt }}$, given by the splitting $\mathcal{A}\left(\operatorname{Ad}_{G}(G)\right)=$ $(A \times T) \ltimes U$, where $A$ is a maximal $\mathbb{R}$-split torus and $U$ is the unipotent radical.

Let $S_{\mathrm{cpt}}$ be a subtorus of $T_{\mathrm{cpt}}$ complementary to $S_{\mathrm{cpt}}^{\perp}$ so that $T_{\mathrm{cpt}}=S_{\mathrm{cpt}} \times S_{\mathrm{cpt}}^{\perp}$. Let $\sigma$ be the composition of the homomorphisms:

$$
\sigma: G \stackrel{\mathrm{Ad}}{\longrightarrow} \mathcal{A}\left(\operatorname{Ad}_{G}(G)\right) \stackrel{\text { projection }}{\longrightarrow} T_{\mathrm{cpt}} \stackrel{\text { projection }}{\longrightarrow} S_{\mathrm{cpt}} \stackrel{x \rightarrow x^{-1}}{\longrightarrow} S_{\mathrm{cpt}} .
$$

One may define the nilshadow map:

$$
\Delta: G \rightarrow S_{\mathrm{cpt}} \ltimes G, g \mapsto(\sigma(g), g),
$$

which is not a homomorphism (unless $S_{\mathrm{cpt}}$ is trivial and then $\sigma$ is trivial), but one has

$$
\Delta(a b)=\Delta\left(\sigma\left(b^{-1}\right) a \sigma(b)\right) \Delta(b), \quad \forall a, b \in G
$$

and $\Delta(\gamma g)=\gamma \Delta(g)$, for every $\gamma \in \tilde{\Gamma}, g \in G$. The nilshadow map $\Delta$ is a diffeomorphism onto its image and so $\Delta(G)$ is simply connected. The product in $\Delta(G)$ is given by:

$$
\Delta(a) \Delta(b)=(\sigma(a), a)(\sigma(b), b)=\left(\sigma(a) \sigma(b), \sigma\left(b^{-1}\right) a \sigma(b) b\right),
$$

for any $a, b \in G$. fore

By construction $\mathcal{A}\left(\operatorname{Ad}_{G}(\tilde{\Gamma})\right)$ projects trivially onto $S_{\text {cpt }}$ and $\sigma(\tilde{\Gamma})=\{e\}$. There-

$$
\tilde{\Gamma}=\Delta(\tilde{\Gamma}) \subset \Delta(G) .
$$

Let $\tilde{G}=\Delta(G)$. By [35, Proposition 4.10] $S_{\text {cpt }}^{\perp}$ is a maximal compact subgroup of $\mathcal{A}\left(\operatorname{Ad}_{\tilde{G}}(\tilde{G})\right)$ and $S_{\text {cpt }}^{\perp} \subset \mathcal{A}\left(\operatorname{Ad}_{\tilde{G}}(\tilde{\Gamma})\right)$, therefore one has $\mathcal{A}\left(\operatorname{Ad}_{\tilde{G}}(\tilde{G})\right)=\mathcal{A}\left(\operatorname{Ad}_{\tilde{G}}(\tilde{\Gamma})\right)$ as shown in [35].

By using Theorem 4.1 we have that $G / \tilde{\Gamma}$ is diffeomorphic to $\tilde{G} / \tilde{\Gamma}$. Finally, by applying Theorem 4.4 we have that $H^{*}(G / \tilde{\Gamma}) \cong H^{*}(\tilde{\mathfrak{g}})$. By considering the diffeomorphism

$$
\Delta: G \rightarrow \tilde{G}
$$

we have that $\Delta^{-1}$ induces a finite-to-one covering map

$$
\Delta^{*}: \tilde{G} / \tilde{\Gamma} \rightarrow G / \Gamma \text {. }
$$

Corollary 6.1. At the level of Lie algebras if we denote by $X_{\mathfrak{s}}$ the image $\sigma_{*}(X)$, for $X \in \mathfrak{g}$, then the Lie algebra $\tilde{\mathfrak{g}}$ of $\tilde{G}$ can be identified by

$$
\tilde{\mathfrak{g}}=\left\{\left(X_{\mathfrak{s}}, X\right): X \in \mathfrak{g}\right\}
$$

with Lie bracket:

$$
\left[\left(X_{\mathfrak{s}}, X\right),\left(Y_{\mathfrak{s}}, Y\right)\right]=\left(0,[X, Y]-\operatorname{ad}\left(X_{\mathfrak{s}}\right)(Y)+\operatorname{ad}\left(Y_{\mathfrak{s}}\right)(X)\right) .
$$

We now obtain some applications of the main theorem by explicitly computing the Lie group $\tilde{G}$. 
Example 6.2 (Nakamura manifold). Consider the simply connected complex solvable Lie group $G$ defined by

$$
G=\left\{\left(\begin{array}{cccc}
e^{z} & 0 & 0 & w_{1} \\
0 & e^{-z} & 0 & w_{2} \\
0 & 0 & 1 & z \\
0 & 0 & 0 & 1
\end{array}\right) \mid w_{1}, w_{2}, z \in \mathbb{C}\right\}
$$

The Lie group $G$ is the semi-direct product $\mathbb{C} \ltimes_{\varphi} \mathbb{C}^{2}$, where

$$
\varphi(z)=\left(\begin{array}{cc}
e^{z} & 0 \\
0 & e^{-z}
\end{array}\right) .
$$

A basis of complex left-invariant 1-forms is given by

$$
\phi_{1}=d z, \phi_{2}=e^{-z} d w_{1}, \phi_{3}=e^{z} d w_{2}
$$

and in terms of the real basis of left-invariant 1 -forms $\left(e^{1}, \ldots, e^{6}\right)$ defined by

$$
\phi_{1}=e^{1}+i e^{2}, \phi_{2}=e^{3}+i e^{4}, \phi_{3}=e^{5}+i e^{6},
$$

we obtain the structure equations:

$$
\left\{\begin{array}{l}
d e^{j}=0, \quad j=1,2 \\
d e^{3}=-e^{13}+e^{24} \\
d e^{4}=-e^{14}-e^{23} \\
d e^{5}=e^{15}-e^{26} \\
d e^{6}=e^{16}+e^{25}
\end{array}\right.
$$

where we denote by $e^{i j}$ the wedge product $e^{i} \wedge e^{j}$.

Let $B \in \operatorname{SL}(2, \mathbb{Z})$ be a unimodular matrix with distinct real eigenvalues: $\lambda, \frac{1}{\lambda}$. Consider $t_{0}=\log \lambda$, i.e. $e^{t_{0}}=\lambda$. Then there exists a matrix $P \in \operatorname{GL}(2, \mathbb{R})$ such that

$$
P B P^{-1}=\left(\begin{array}{cc}
\lambda & 0 \\
0 & \lambda^{-1}
\end{array}\right) \text {. }
$$

Let

$$
\begin{aligned}
& L_{1,2 \pi}=\mathbb{Z}\left[t_{0}, 2 \pi i\right]=\left\{t_{0} k+2 \pi h i, h, k \in \mathbb{Z}\right\}, \\
& L_{2}=\left\{P\left(\begin{array}{c}
\mu \\
\alpha
\end{array}\right) \mid \mu, \alpha \in \mathbb{Z}[i]\right\} .
\end{aligned}
$$

Then, by [33] $\Gamma=L_{1,2 \pi} \ltimes_{\varphi} L_{2}$ is a lattice of $G$.

Since $G$ has trivial center, we have that $\operatorname{Ad}_{G}(G) \cong G$ and thus it is a semidirect product $\mathbb{R}^{2} \ltimes \mathbb{R}^{4}$. Moreover, for the algebraic closures of $\operatorname{Ad}_{G}(G)$ and $\operatorname{Ad}_{G}(\Gamma)$ we obtain

$$
\mathcal{A}\left(\operatorname{Ad}_{G} G\right)=\left(\mathbb{R}^{\#} \times S^{1}\right) \ltimes \mathbb{R}^{4}, \quad \mathcal{A}\left(\operatorname{Ad}_{G} \Gamma\right)=\mathbb{R}^{\#} \ltimes \mathbb{R}^{4},
$$


where the split torus $\mathbb{R}^{\#}$ corresponds to the action of $e^{\frac{1}{2}(z+\bar{z})}$ and the compact torus $S^{1}$ to the one of $e^{\frac{1}{2}(z-\bar{z})}$.

Therefore in this case $\mathcal{A}\left(\operatorname{Ad}_{G}(G)\right)=S^{1} \mathcal{A}\left(\operatorname{Ad}_{G}(\Gamma)\right)$ and $\mathcal{A}\left(\operatorname{Ad}_{G}(\Gamma)\right)$ is connected. By applying the main theorem there exists a simply connected normal subgroup $\tilde{G}=\Delta(G)$ of $S^{1} \ltimes G$. The new Lie group $\tilde{G}$ is obtained by killing the action of $e^{\frac{1}{2}(z-\bar{z})}$. Indeed, we get that

$$
\tilde{G} \cong\left\{\left(\begin{array}{cccc}
e^{\frac{1}{2}(z+\bar{z})} & 0 & 0 & w_{1} \\
0 & e^{-\frac{1}{2}(z+\bar{z})} & 0 & w_{2} \\
0 & 0 & 1 & z \\
0 & 0 & 0 & 1
\end{array}\right) \mid w_{1}, w_{2}, z \in \mathbb{C}\right\}
$$

The diffeomorphism between $G / \Gamma$ and $\tilde{G} / \Gamma$ was already shown in [33]. Then in this case one has the isomorphism $H_{\mathrm{dR}}^{*}(G / \Gamma) \cong H^{*}(\tilde{\mathfrak{g}})$, where $\tilde{\mathfrak{g}}$ denotes the Lie algebra of $\tilde{G}$ and the de Rham cohomology of the Nakamura manifold $G / \Gamma$ is not isomorphic to $H^{*}(\mathfrak{g})$ (see also [9]).

Example 6.3. Let consider the 3-dimensional solvable Lie group $\mathbb{R} \ltimes \mathbb{R}^{2}$ with structure equations

$$
\left\{\begin{array}{l}
d e^{1}=0 \\
d e^{2}=2 \pi e^{13} \\
d e^{3}=-2 \pi e^{12} .
\end{array}\right.
$$

is a non-completely solvable Lie group which admits a compact quotient and the uniform discrete subgroup is of the form $\Gamma=\mathbb{Z} \ltimes \mathbb{Z}^{2}$ (see [29, Theorem 1.9] and [24]). Indeed, the Lie group $\mathbb{R} \ltimes \mathbb{R}^{2}$ is the group of matrices

$$
\left(\begin{array}{cccc}
\cos (2 \pi t) & \sin (2 \pi t) & 0 & x \\
-\sin (2 \pi t) & \cos (2 \pi t) & 0 & y \\
0 & 0 & 1 & t \\
0 & 0 & 0 & 1
\end{array}\right)
$$

and the lattice $\Gamma$ generated by 1 in $\mathbb{R}$ and the standard lattice $\mathbb{Z}^{2}$, as in Example 4.2.

By applying the main theorem we have that $\mathcal{A}\left(\operatorname{Ad}_{G}(G)\right)=S^{1} \ltimes \mathbb{R}^{2}$ and $\mathcal{A}\left(\operatorname{Ad}_{G}(\Gamma)\right)=\mathbb{R}^{2}$. Therefore in this case $\tilde{G} \cong \mathbb{R}^{3} \subset S^{1} \ltimes G$. By applying the main theorem we get that $\tilde{G} \cong \mathbb{R}^{3}$. Indeed, it is well known that $G / \Gamma$ is diffeomorphic to a torus.

The previous example $\mathbb{R} \ltimes \mathbb{R}^{2}$ is a special case of an almost Abelian Lie group. We recall that a Lie algebra $\mathfrak{g}$ is called almost Abelian if it has an Abelian ideal of codimension 1 , i.e. if it can be represented as a semidirect sum $\mathbb{R} \ltimes \mathfrak{b}$, where $\mathfrak{b}$ is an Abelian ideal of $\mathfrak{g}$. In [13] Gorbatsevich found a sufficient and necessary condition under which given an almost Abelian Lie group $G$ and a lattice $\Gamma$ of $G$ one has the equality $\mathcal{A}\left(\operatorname{Ad}_{G} G\right)=\mathcal{A}\left(\operatorname{Ad}_{G} \Gamma\right)$. More precisely, he showed the following: 
Theorem 6.4 ([13, Theorem 4]). Let $G=\mathbb{R} \ltimes_{\varphi} \mathbb{R}^{n}$ be a simply connected almost Abelian Lie group and $\Gamma=\mathbb{Z} \ltimes \mathbb{Z}^{n}$ be a lattice in $G$. Let $z$ be a generator for the subgroup $\mathbb{Z} \subset \Gamma$. The action of $z$ on $\mathbb{R}^{n}$ defines some matrix, i.e., $z \in \mathrm{GL}(\mathfrak{g})$ and suppose that the one-parameter subgroup corresponding to the subgroup $A=\mathbb{R}$ is $\exp (t \cdot Z)$, where $Z$ is some matrix in the Lie algebra of derivations of the Lie algebra $\mathfrak{n}$ of the nilradical $N$ of $G$. Therefore there is a unipotent matrix $J$ such that $z=J \cdot \exp (Z)$.

Then $\mathcal{A}\left(\operatorname{Ad}_{G} G\right)=\mathcal{A}\left(\operatorname{Ad}_{G} \Gamma\right)$ if and only if the number $i \pi$ is not representable as a linear combination of the numbers $\lambda_{k}, k=1, \ldots, n$, with rational coefficients, where $\left\{\lambda_{1}, \ldots, \lambda_{n}\right\}$ is the spectrum of $Z$.

The idea for the proof of the previous theorem is that

$$
\begin{aligned}
& \mathcal{A}\left(\operatorname{Ad}_{G}(G)\right)=\mathcal{A}\left(\operatorname{Ad}_{G}(A)\right) \ltimes \operatorname{Ad}_{G}(N), \\
& \mathcal{A}\left(\operatorname{Ad}_{G}(\Gamma)\right)=\mathcal{A}\left(\operatorname{Ad}_{G}(\mathbb{Z})\right) \ltimes \operatorname{Ad}_{G}(N),
\end{aligned}
$$

since $\operatorname{Ad}_{G}(N)$ is algebraic closed. Therefore, finding $\mathcal{A}\left(\operatorname{Ad}_{G}(\Gamma)\right)$ reduces to finding the algebraic closure of the image of a cyclic subgroup (i.e. $\mathbb{Z}$ in the above decomposition for $\Gamma)$. In comparing $\mathcal{A}\left(\operatorname{Ad}_{G}(A)\right)$ and $\mathcal{A}\left(\operatorname{Ad}_{G}(\mathbb{Z})\right)$ the unipotent matrix $J$ plays no role, so one can suppose that $z=\exp (Z)$. Therefore, we may assume that $A=\exp (t Z)$ is the one-parameter subgroup generated by $Z$ and $\mathbb{Z}$ is the cyclic subgroup generated by $z=\exp (Z)$. One can use the decomposition $z=$ $z_{s} z_{u}$, where $z_{s}$ (the semisimple part) is diagonal, i.e. $z_{s}=\operatorname{diag}\left(\exp \left(\lambda_{1}, \ldots, \lambda_{n}\right)\right)$ and $z_{u}$ is unipotent. The semisimple part of the algebraic closure of $\mathbb{Z}$ consists of diagonal matrices and then it is contained in some algebraic torus. It is also clear that the algebraic closure of the semisimple part $z_{s}$ is the intersection of the kernels of the diagonal matrices $\Pi_{k} e^{\lambda_{k} n_{k}}$.

In this way, Gorbatsevich showed that $\mathcal{A}\left(\operatorname{Ad}_{G}(A)\right)=\mathcal{A}\left(\operatorname{Ad}_{G}(\mathbb{Z})\right)$ if and only if $i \pi$ is not representable as a linear combination of the numbers $\lambda_{k}, k=1, \ldots, n$, with rational coefficients. If $i \pi$ is not representable as a linear combination of the numbers $\lambda_{k}, k=1, \ldots, n$, with rational coefficients, then one may apply Theorem 4.4 to compute the de Rham cohomology of the compact solvmanifold $G / \Gamma$ and one has that $H_{\mathrm{dR}}^{*}(G / \Gamma) \cong H^{*}(\mathfrak{g})$. Otherwise, i.e. if $i \pi$ is a rational linear combination of the numbers $\lambda_{k}, k=1, \ldots, n$, the only known result about the de Rham cohomology of $G / \Gamma$ is that (see [5, Proposition 4.7])

$$
b_{1}(G / \Gamma)=n+1-\operatorname{rank}(\varphi(1)-i d) .
$$

By applying the main theorem one obtains a method to compute the de Rham cohomology of $G / \Gamma$ even in the latter case. It is not restrictive to suppose that $\mathcal{A}\left(\operatorname{Ad}_{G} \Gamma\right)$ is connected (otherwise one considers a finite index subgroup $\tilde{\Gamma}$ of $\Gamma$ ). Then $\mathcal{A}\left(\operatorname{Ad}_{G} G\right)=S^{1} \mathcal{A}\left(\operatorname{Ad}_{G} \Gamma\right)$, or equivalently $\mathcal{A}\left(\operatorname{Ad}_{G}(A)\right)=S^{1}$, and we may apply the main theorem.

Example 6.5. (Hyperelliptic surface) Consider the solvable Lie group $G=\mathbb{R} \ltimes_{\varphi}$ $(\mathbb{C} \times \mathbb{R})$, where the action $\varphi: \mathbb{R} \rightarrow \operatorname{Aut}(\mathbb{C} \times \mathbb{R})$ is defined by

$$
\varphi(t)(z, s)=\left(e^{i \eta t} z, s\right)
$$


where $\eta=\pi, \frac{2}{3} \pi, \frac{1}{2} \pi$ or $\frac{1}{3} \pi$. By [18] $G$ has seven isomorphism classes of lattices $\Gamma=\mathbb{Z} \ltimes_{\varphi} \mathbb{Z}^{3}$ where the action $\varphi: \mathbb{Z} \rightarrow \operatorname{Aut}\left(\mathbb{Z}^{3}\right)$ is defined by a matrix $\varphi(1)$ which has eigenvalues $1, e^{i \eta}$ and $e^{-i \eta}$. Since $\varphi(1)$ has a pair of complex conjugate imaginary roots, by applying Theorem 6.4 , in this case we have $\mathcal{A}\left(\operatorname{Ad}_{G}(G)\right) \neq$ $\mathcal{A}\left(\operatorname{Ad}_{G}(\Gamma)\right)$. In this case $\mathcal{A}\left(\operatorname{Ad}_{G}(\Gamma)\right)$ is not connected, but we have that $\Gamma$ contains as a finite index subgroup $\tilde{\Gamma} \cong \mathbb{Z}^{4}$. In this way we get that the compact solvmanifold $G / \Gamma$ is a finite covering of a torus. Note that in this case $H_{\mathrm{dR}}^{1}(G / \Gamma) \cong H^{1}(\mathfrak{g})$ even if $G$ and $\Gamma$ do not satisfy the Mostow condition. Indeed, $G$ has structure equations

$$
\left\{\begin{array}{l}
d e^{1}=e^{24} \\
d e^{2}=-e^{14} \\
d e^{3}=0 \\
d e^{4}=0
\end{array}\right.
$$

and $H^{1}(\mathfrak{g})=\operatorname{span}\left\langle e^{3}, e^{4}\right\rangle$.

\section{References}

[1] L. AUSLANDER, An exposition of the structure of solvmanidolds I and II, Bull. Amer. Math. Soc. 79 (1973), 227-261.

[2] L. Auslander and J. Brezin, Almost algebraic Lie algebras, J. Algebra 8 (1968), 295313.

[3] L. Auslander and R. TOlimieri, Splitting theorems and the structure of solvmanifolds, Ann. of Math. (2) 92 (1970), 164-173.

[4] O. BAUES and V. CORTES, Aspherical Kähler manifolds with solvable fundamental group, Geom. Dedicata 122 (2006), 215-229.

[5] C. Bock, On low-dimensional solvmanifolds, preprint arXiv:0903.2926 (2009).

[6] A. Borel, "Linear Algebraic Groups", Second edition, Graduate Texts in Mathematics, Vol. 126, Springer, New York, 1991.

[7] S. CONSOLE and A. FINO, Dolbeault cohomology of compact nilmanifolds, Transform. Groups 6 (2001), 111-124.

[8] A. Cordero, M. Fernández, A. Gray and L. Ugarte, Compact nilmanifolds with nilpotent complex structures: Dolbeault cohomology, Trans. Amer. Math. Soc. 352 (2000), $5405-5433$.

[9] P. DE BARTOlomeIs and A. Tomassini, On solvable generalized Calabi-Yau manifolds, Ann. Inst. Fourier (Grenoble) 56 (2006), 1281-1296.

[10] K. DEKIMPE, Semi-simple splitting for solvable Lie groups and polynomial structures, Forum Math. 12 (2000), 77-96.

[11] V.V. GorbatSEVICH, Splittings of Lie groups and their application to the study of homogeneous spaces, Math. USSR-Izv. 15 (1980), 441-467.

[12] V.V. GoRbATSEVICH, Plesicompact homogeneous spaces, Siber. Math. J. 30 (1989), $217-$ 226.

[13] V.V. Gorbatsevich, Symplectic structures and cohomologies on some solvmanifolds, Siber. Math. J. 44 (2003), 260-274.

[14] W. Greub, S. Halperin and R. Vanstone, "Connections, Curvature and Cohomology", Vol. I, Academic Press, New York and London, 1973.

[15] D. GUAN, Modification and the cohomology groups of compact solvmanifolds, Electron. Res. Announc. Amer. Math. Soc. 13 (2007), 74-81. 
[16] D. GuAn, Classification of compact complex homogeneous manifolds with pseudoKŁhlerian structures, J. Algebra 324 (2010), 2010-2024.

[17] D. GUAN, Classification of compact homogeneous manifolds with pseudo-Kählerian structures, C. R. Math. Acad. Sci. Soc. R. Can. 31 (2009), 20-23.

[18] K. Hasegawa, Complex and Kähler structures on compact solvmanifolds, J. Symplectic Geom. 3 (2005), 749-767.

[19] A. Hattori, Spectral sequence in the de Rham cohomology of fibre bundles, J. Fac. Sci. Univ. Tokyo Sect. I 8 (1960), 289-331.

[20] A. Malcev, On solvable Lie algebras, Bull. Acad. Sci. URSS. Sér. Math. [Izvestia Akad. Nauk SSSR] 9 (1945), 329-356.

[21] Y. I. MerziLyakov, "Rational Groups", Nauka, Moscow, 1987.

[22] J. Milnor, Curvature of left invariant metrics on Lie groups, Adv. Math. 21 (1976), 293329.

[23] M. V. Milovanov, Description of solvable Lie groups with a given uniform subgroup, Mat. Sb. (N.S.) 113(155) (1980), 98-117, 175.

[24] D. V. MillionsCHIKOV, Multivalued functionals, one-forms and deformed de Rham complex, e-print math.AT/0512572 (2005).

[25] G. Mostow, Factor spaces of solvable spaces, Ann. of Math. (2) 60 (1954), 1-27.

[26] G. Mostow, Cohomology of topological groups and solvmanifolds, Ann. of Math. (2) 73 (1961), 20-48.

[27] K. NomizU, On the cohomology of homogeneous spaces of nilpotent Lie Groups, Ann. of Math. (2) 59 (1954), 531-538.

[28] A. L. OnishchiK and E. B. Vinberg, "Lie Groups and Lie Algebras II. Discrete Subgroups of Lie Groups and Cohomologies of Lie Groups and Lie Algebras", Encyclopaedia of Mathematical Sciences, Vol. 21, Springer-Verlag, Berlin, 2000.

[29] J. Oprea and A. Tralle, "Symplectic Manifolds with no Kähler Structure", Lecture Notes in Mathematics, Vol. 1661, Springer, Berlin, 1997.

[30] M. S. Raghunathan, "Discrete subgroups of Lie groups", Springer, Berlin, 1972.

[31] S. RollensKe, Lie-algebra Dolbeault-cohomology and small deformations of nilmanifolds, J. London Math. Soc. (2) 79 (2009), 346-362.

[32] A. N. STARKOV, "Algebraic Groups and Homogeneous Spaces of Finite Volume", Translated from the 1999 Russian original by the author, Translations of Mathematical Monographs, Vol. 190, American Mathematical Society, Providence, RI, 2000.

[33] T. YAMADA, A pseudo-Kähler structure on a nontoral compact complex parallelizable solvmanifold, Geom. Dedicata 112 (2005), 115-122.

[34] D. WiTTE, Zero-entropy affine maps on homogeneous spaces, Amer. J. Math. 109 (1987), 927-961.

[35] D. WITTE, Superrigidity of lattices in solvable Lie groups, Invent. Math. 122 (1995), 147193.

Dipartimento di Matematica G. Peano Università di Torino

Via Carlo Alberto, 10

10123 Torino, Italia sergio.console@unito.it annamaria.fino@unito.it 